\title{
Evolution of anisotropic microstructure and residual stress in sputtered $\mathrm{Cr}$ films
}

\author{
Z. B. Zhao a) \\ Delphi Research Laboratories, 51786 Shelby Parkway, Shelby Township, Michigan 48315 \\ S. M. Yalisove \\ Center for Nanomaterials Science, Department of Materials Science and Engineering, \\ The University of Michigan, Ann Arbor, Michigan 48109-2136 \\ Z. U. Rek \\ Stanford Synchrotron Radiation Laboratory, Stanford University, California 94309 \\ J. C. Bilello \\ Center for Nanomaterials Science, Department of Materials Science and Engineering, \\ The University of Michigan, Ann Arbor, Michigan 48109-2136
}

(Received 11 June 2002; accepted 24 September 2002)

\begin{abstract}
A series of $\mathrm{Cr}$ films with varying thicknesses have been prepared using a multiple moving substrate deposition geometry. These films have been investigated with several experimental techniques, including synchrotron $\mathrm{x}$-ray scattering, pole figures, electron microscope, and double crystal diffraction topography. It was found that the in-plane stresses are highly anisotropic in these $\mathrm{Cr}$ films. The anisotropic stresses, characterized by two principal stresses in two characteristic directions defined by the deposition geometry, are quantified based on a methodology given in the Appendix. The plan view transmission electron microscopy observations reveal that the $\mathrm{Cr}$ films develop well-organized microstructures. The grains, which are elongated along the radial direction, are crystallographically aligned as well. The development of crystallographic texture in the $\mathrm{Cr}$ films, further revealed by pole figures and azimuthal $(\phi)$ x-ray scans, depends on both the deposition geometry and the film thickness. The preferential orientation of film growth is [110] for thinner films $(<1.6 \mu \mathrm{m})$, and then becomes [111] for thicker films. Correspondingly, the in-plane texture varies in a conformal manner. In the former case, [100] and [110] directions of grains preferentially align along the radial direction and the direction of platen rotation, respectively. In the latter case, the preferential orientation of grains in the radial direction becomes [112], while that in the direction of rotation remains to be [110]. The occurrence of the anisotropic stresses and their dependence on film thickness is related to the evolution of the anisotropic structure and in-plane texture. The correlation is discussed in terms of the modulus effect associated with in-plane texture, the stress relief at intercolumnar voids, and the texture transition. (C) 2002 American Institute of Physics.
\end{abstract}

[DOI: 10.1063/1.1521791]

\section{INTRODUCTION}

$\mathrm{Cr}$ films are used as engineering protective coatings for wear and corrosion resistance, ${ }^{1}$ also used in photomasks, integrated circuits, optical, and magnetic devices. ${ }^{2-4}$ For most applications, the film properties and performance strongly depend on the crystallographic orientation (texture) of the grains in the films. ${ }^{5,6}$ For example, Cr films, used as an underlayer for thin magnetic recording media, have been shown to significantly enhance the coercivity of Co and Co-based magnetic films if its crystallographic orientation is properly controlled. ${ }^{7,8}$ The preferred orientations of magnetron sputtered Cr films are reported to be either (110) or (200), depending on Ar pressure, substrate temperature, and substrate bias. ${ }^{2,3,7,8}$ Ravipati et al. have observed (110) texture in the Cr films deposited at low-argon pressure and low-substrate temperature, and (200) texture in the films deposited at high

\footnotetext{
a)Electronic mail: zhibo.zhao@delphiauto.com
}

temperatures. ${ }^{9}$ Tsai et al. have studied $\mathrm{Cr}$ films as the underlayer for Co-based films, and have indicated that development of (110) or (200) textures of Cr film is closely related to the types of substrate materials. ${ }^{10}$ This result, however, is not supported by the recent observations made by Miller and Holland, ${ }^{4}$ Tang and Thomas, ${ }^{3}$ and Feng et al. ${ }^{8}$ These authors have indicated that the preheating of substrate appears to be the deciding factor for forming (200) texture, regardless of what types of substrates are used. Two mechanisms for texture formation have been proposed by Feng et al. for $\mathrm{Cr}$ films: ${ }^{8}$ (i) The crystallographic texture originates from the preferential orientations of islands before continuous film forms and (ii) the crystallographic texture develops due to faster growth of grains with favorable orientations after the films become a continuous film. The authors contributed the former mechanism to the (110) texture and the latter to the (200) texture. Although not stated explicitly, these studies on $\mathrm{Cr}$ have focused primarily on out-of-plane texture, i.e., the crystallographic alignments in the direction of film growth. 




FIG. 1. The multiple moving substrate deposition geometry used for the magnetron sputtered $\mathrm{Cr}$ films. Note that the multiple substrates move underneath the cathode through the rotation of a support plate.

Of particular relevance to this work are the observations by Karpenko et al. who reported that the sputter-deposited Mo thin films (under certain deposition geometry) can develop in-plane texture. ${ }^{11}$ The authors have proposed that selfshadowing effect and the anisotropic shape growth of grains are primarily responsible for the development of the in-plane texture. ${ }^{12}$ However, whether it is a general phenomenon for deposited materials or just unique for Mo films is unknown. This work is intended to address this issue through the structural characterization of magnetron sputtered $\mathrm{Cr}$ films.

Generally, $\mathrm{Cr}$ is considered to have low-atomic mobility. ${ }^{13}$ Thus, vapor-deposited $\mathrm{Cr}$ films tend to develop large tensile residual stress, which often affect their adhesion to substrates. ${ }^{1}$ Many studies ${ }^{14-17}$ have revealed the direct link between the microstructure and the stress state in thin films. One objective of the present study is to determine the stress states of the Cr films and to identify their correlation to the film structures. The emphasis will be placed on the evolution of the in-plane anisotropic characteristics of the films.

\section{SPECIMEN PREPARATIONS AND EXPERIMENTS}

A series of Cr films with varying thickness $(0.1-4.5 \mu \mathrm{m})$ were prepared using dc magnetron sputtering, with a multiple moving substrate geometry shown in Fig. 1. In such a geometry, four Si (100) single crystal wafers were placed onto a $\phi=30-\mathrm{cm}$ rotation platen, and the center of each substrate is about $25 \mathrm{~cm}$ from the rotation axis. The $\mathrm{Si}$ substrates were always oriented in a consistent manner such that their [110] flat edges align with the radial direction of the platen. The chamber base pressure was typically less than 1 $\times 10^{-6}$ Torr. Prior to deposition, the Cr target was presputtered for $5 \mathrm{~min}$ to remove the surface oxides and thus reduce

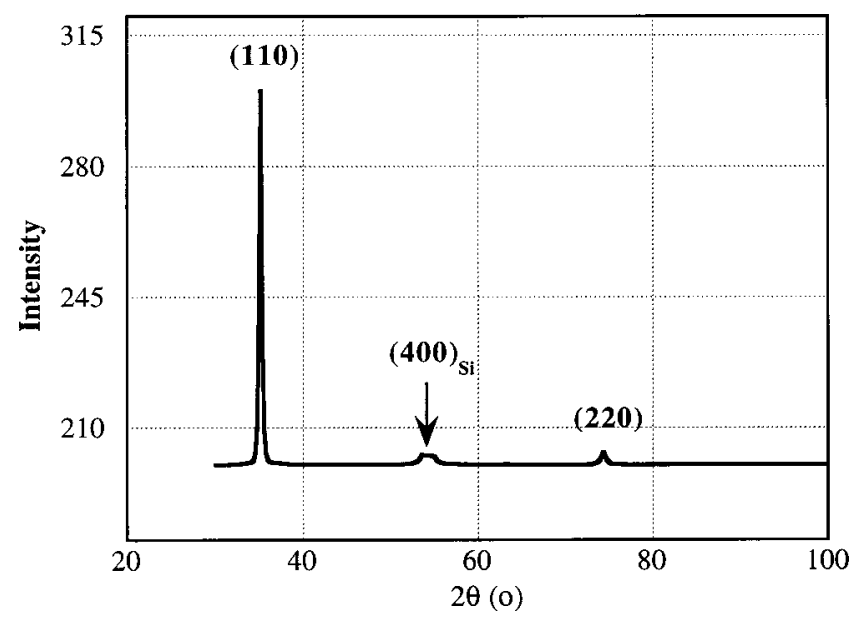

FIG. 2. The X-ray diffraction pattern of a 1.6- $\mu \mathrm{m} \mathrm{Cr}$ film obtained with symmetric reflection geometry, which indicates the (110) type out-of-plane texture.

the impurities in the films. The primary deposition parameters include 460-W power, 2-mTorr Ar pressure, and 6-cm target-substrate distance.

The x-ray scattering experiments were conducted at the Stanford Synchrotron Radiation Laboratory under standard conditions (3-GeV and $100-\mathrm{mA}$ at fill). The monochromatic $\mathrm{x}$-ray with wavelength $0.124 \mathrm{~nm}$ was selected using a $\mathrm{Si}$ (111) double-crystal monochromator. The wavelength was calibrated via a standard $\mathrm{LaB}_{6}$ powder specimen. The horizontal divergence and vertical divergence of the beam were 3 and $0.2 \mathrm{mrad}$, respectively. The incident beam was defined by a $1 \mathrm{~mm} \times 1 \mathrm{~mm}$ slit. Specimens were mounted onto a Huber 5020 four-circle diffractometer. The [110] flat edges of (100) Si substrates were used as a reference so that all
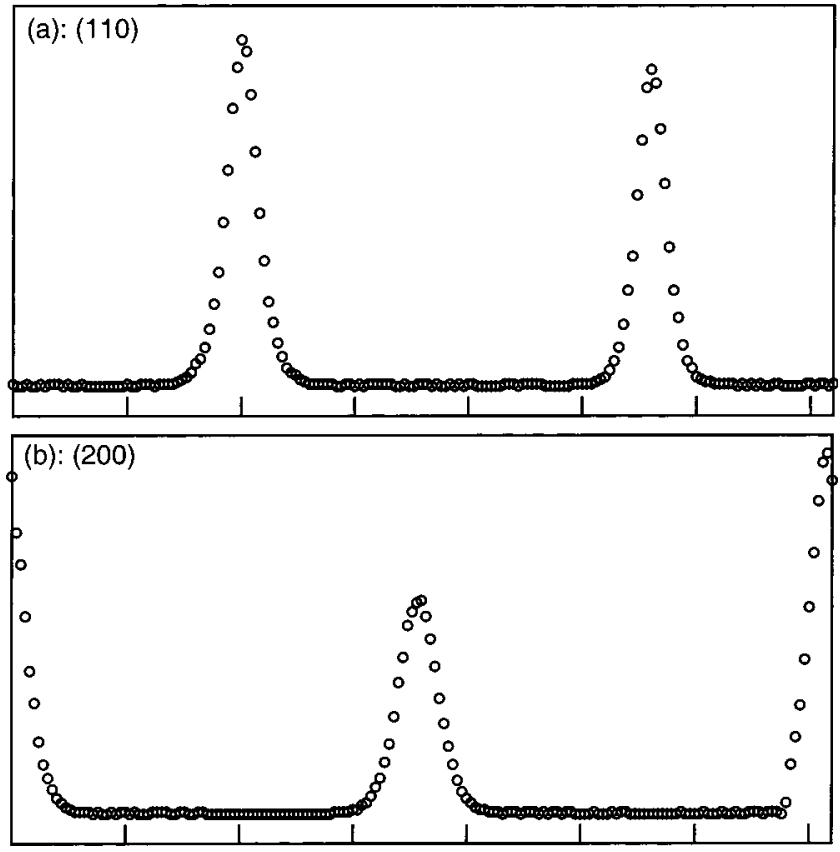

FIG. 3. Intensity distribution of the (110) and (200) reflections from a $1.6-\mu \mathrm{m} \mathrm{Cr}$ film, obtained by azimuthal $\phi$ scan at a grazing incidence angle obtained by azimuthal $\phi$ scan at a grazing incidence angle. 
(a)

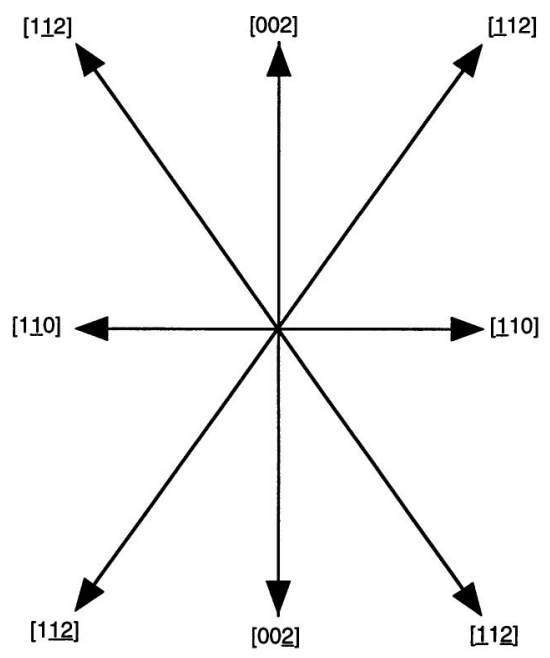

(b)

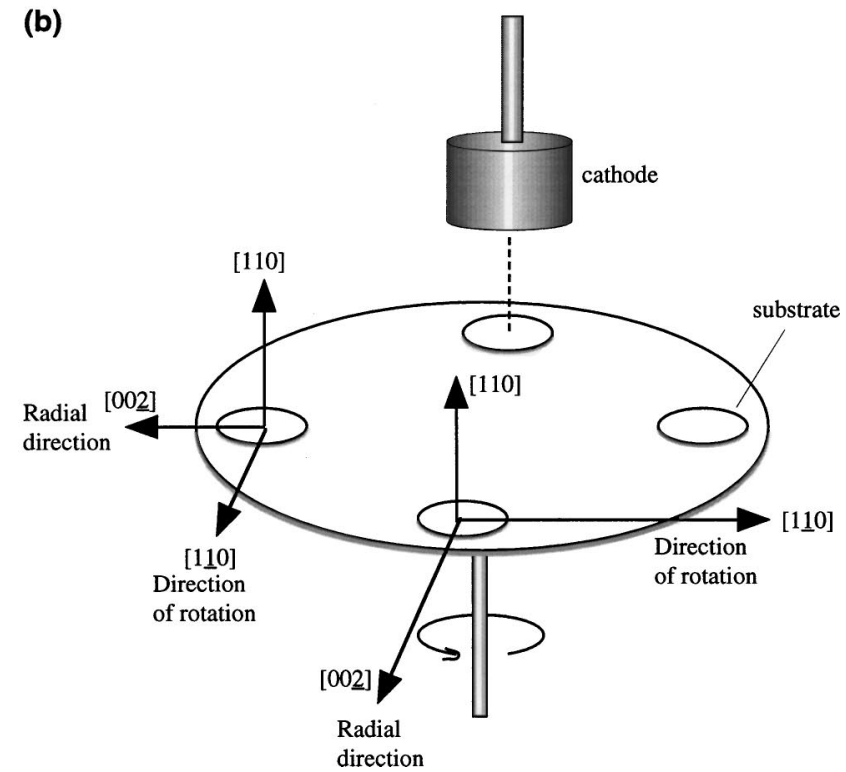

FIG. 4. Intensity distribution of the (110) and (200) reflections from a 1.6- $\mu \mathrm{m} \mathrm{Cr}$ film, obtained by azimuthal $\phi$ scan at a grazing incidence angle.

specimens were oriented consistently with respect to the incident beam. Diffracted x rays, whose vertical divergence was limited by a 1-mrad Soller slit, were received by a Germanium solid-state detector with nitrogen cooling. The symmetric reflection geometry was used for determination of the out-of-plane texture. The symmetric grazing incidence geometry (GIXS) was used to collect the reflection intensities from the lattice planes that are nearly parallel to the film normal. The Bragg conditions for major reflections of each Cr film such as (110), (200), and (211) were first established. Then azimuthal $\phi$ scans were performed, where the intensity of a particular reflection was collected while the specimen was rotated along its normal. Apparently, the diffraction intensity at any $\phi$ is directly proportional to the volume fraction of grains that have this particular in-plane orientation. For a GIXS experiment, usually only the top layer of a film contributes to the diffraction intensity. Such a layer is estimated to be $\sim 100$-nm thick for all the Cr films in this study.

Surface morphologies of $\mathrm{Cr}$ films were examined with a scanning electron microscope. Microstructural observations
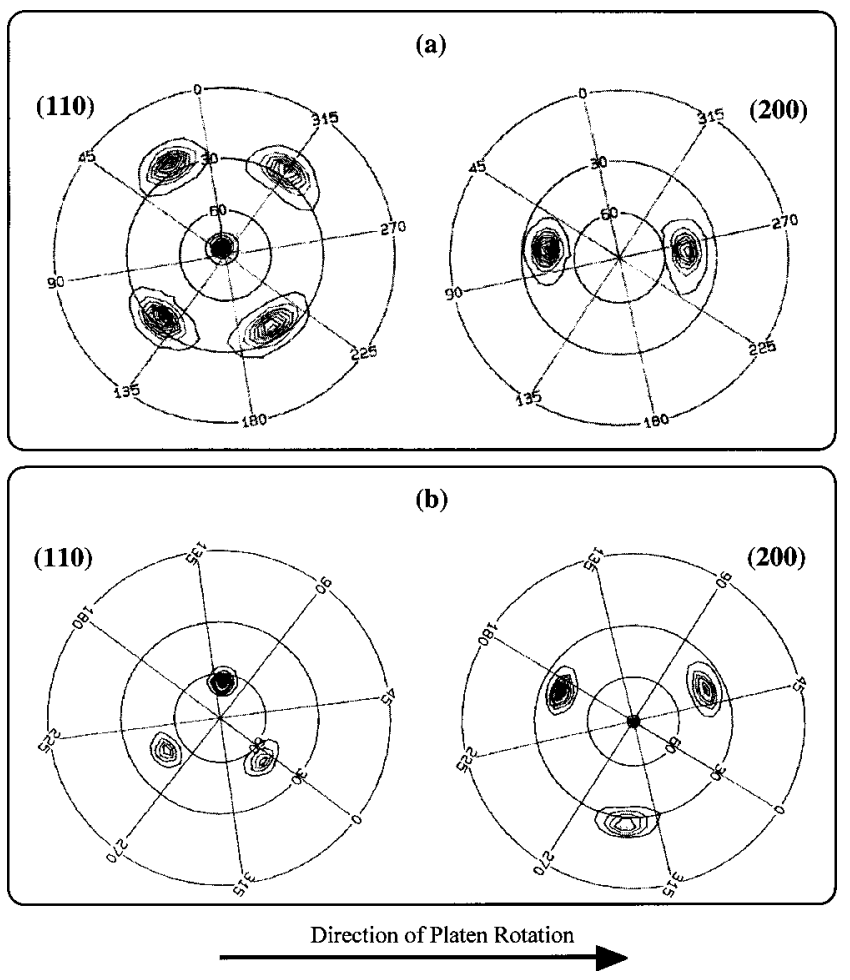

FIG. 5. Pole figures of two Cr films (a) $1.6-\mu \mathrm{m}$ thick and (b) $4.5-\mu \mathrm{m}$ thick.

were performed on selected $\mathrm{Cr}$ films with transmission electron microscopy. To prepare TEM thin foils, 3-mm discs were prepared by ultrasonic cutting and mechanically thinned from the substrate side to $\sim 125 \mu \mathrm{m}$. The discs were then chemically etched using a $\mathrm{HNO}_{3}: \mathrm{HF}: \mathrm{CH}_{3} \mathrm{COOH}$ mixture (3:5:3) until $\mathrm{Cr}$ films became partially exposed. Subsequently, the specimens were ion milled at $5 \mathrm{KeV}$ either from one side or both sides to achieve electron transparency.

Thin-film texture was measured from reflection x-ray pole figures collected using a Rigaku Rotaflex x-ray system equipped with a $12-\mathrm{KW}$ rotating anode $(\mathrm{Cu} \mathrm{K} \alpha$ radiation) and a Rigaku texture diffractometer configured in the Schultz geometry.

In order to determine the stresses in $\mathrm{Cr}$ films, all the $\mathrm{Si}$ wafers characterized by double-crystal diffraction topography before and after film deposition. The curvature differentials were used to calculate the stresses in thin films based on the equations given in the Appendix.

\section{RESULTS OF STRUCTURAL CHARACTERIZATION}

\section{A. Structure of the texture in the $1.6-\mu \mathrm{m} \mathrm{Cr}$ film}

Figure 2 shows the diffraction pattern of a 1.6- $\mu \mathrm{m} \mathrm{Cr}$ film, which is obtained using the symmetric reflection geometry. Thus, the appearance of only the (110) reflection indicates that the out-of-plane texture is the (110\} type. The azimuthal $(\phi)$ scans on the same film provide the in-plane intensity distributions of two major reflections of bcc $\mathrm{Cr}$ : (110) and (200), as given in Fig. 3. The presence of the strong peaks in these patterns suggests that the grains have the preferential crystallographic orientations in the plane of the film, namely, the in-plane texture. The structure of tex- 


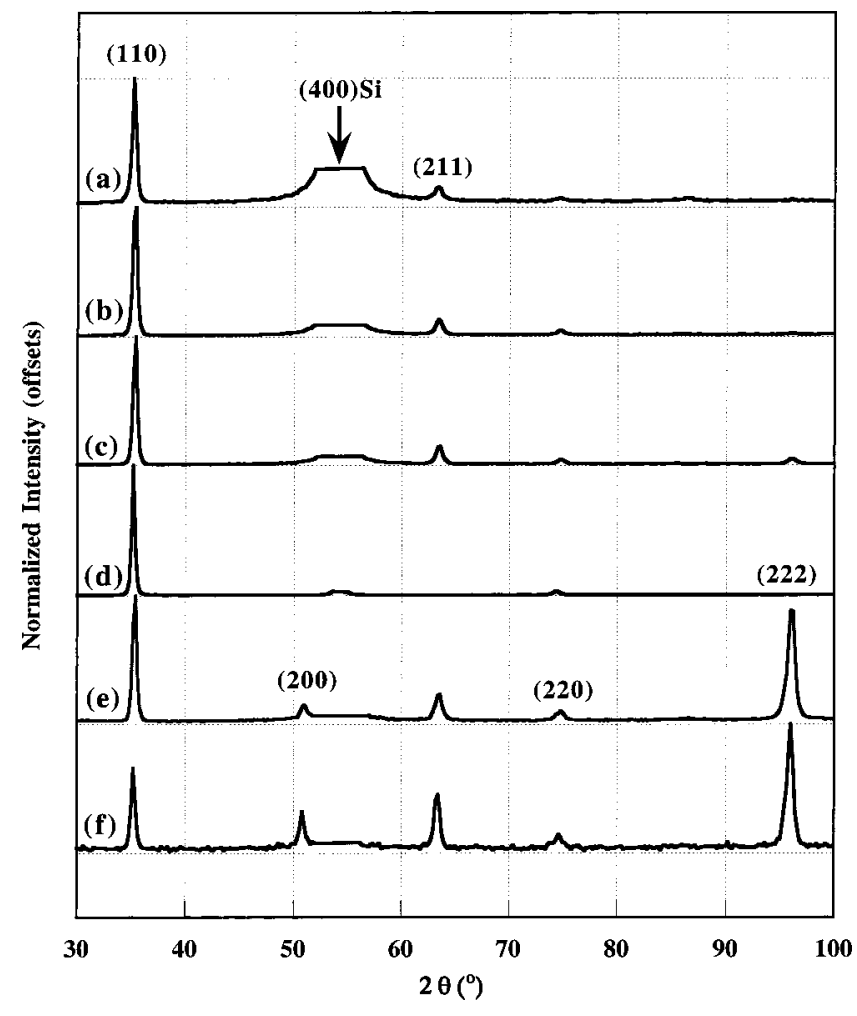

FIG. 6. The x-ray diffraction patterns of $\mathrm{Cr}$ films with different thicknesses, obtained with symmetric reflection geometry. The results indicate that the out-of-plane texture changes from the (110) type to the (111) types after a certain thickness. The nominal thicknesses of the films: (a) $0.1 \mu \mathrm{m}$; (b) 0.4 $\mu \mathrm{m}$; (c) $0.8 \mu \mathrm{m}$; (d) $1.6 \mu \mathrm{m}$; (e) $3.2 \mu \mathrm{m}$; and (e) $4.5 \mu \mathrm{m}$.

ture can be inferred from the patterns shown in Figs. 2 and 3 with aid of the (110) projection [Fig. 4(a)]. Based on the diffraction geometry of $\phi$ scan, one can establish that the structure of the texture is related to the deposition geometry. The result is schematically illustrated in Fig. 4(b). The texture is represented by the preferential crystallographic orientations in three characteristic directions as defined by the deposition geometry, namely, [110], [110], and [100] preferentially align in the direction of film growth, the radial direction, and the direction of platen rotation, respectively. These crystallographic orientations conform to the relationship governed by a cubic lattice. Thus, the texture in the $\mathrm{Cr}$ film is triaxial in nature. Shown in Fig. 5(a) are the (110) and (200) pole figures, which further verifies the structure of texture and its correlation with the deposition geometry, as represented in Fig. 4(b).

\section{B. Thickness dependence of textures in $\mathrm{Cr}$ films}

Figure 6 shows the diffraction patterns of a series of $\mathrm{Cr}$ films with varying thickness, which were obtained via symmetric reflection geometry. For comparison, the strongest peak in each pattern is normalized to $100 \%$. The thinner films $(0.1,0.4$, and $0.8 \mu \mathrm{m})$ exhibit similar diffraction patterns as that of the $1.6-\mu \mathrm{m}$ film, indicating that these films also have the (110)-type out-of-plane texture. However, in the diffraction patterns of thicker films (3.2 and $4.5 \mu \mathrm{m})$, the strong (222) reflection appears in addition to the (110) reflection. Note that for a bulk $\mathrm{Cr}$ sample, the intensity of the

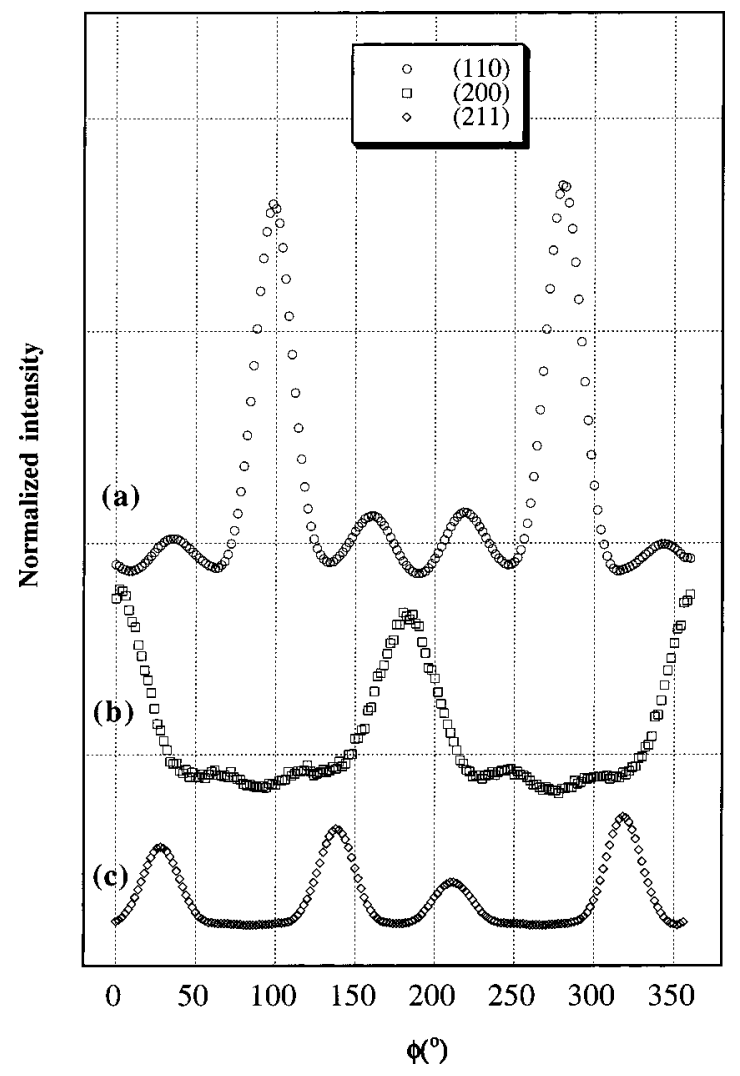

FIG. 7. Intensity distribution of the (110), (200), and (211) reflections from a $0.8-\mu \mathrm{m} \mathrm{Cr}$ film, obtained by azimuthal $\phi$ scan at a grazing incidence angle.

(222) reflection is usually only $6 \%$ of that of the (110) reflection, as reported in the Joint Committee for Powder Diffraction Standard card. Therefore, the diffraction patterns in Figs. 6(e) and 6(f) suggest the occurrence of the (111)-type out-of-plane texture in thicker $\mathrm{Cr}$ films. This implies that the out-of-plane texture changes from the (110) type to (111) type, which occurs between 1.6 and $3.2 \mu \mathrm{m}$.

Figure 7 shows the azimuthal scan results of a $0.8 \mu \mathrm{m} \mathrm{Cr}$ film, as an example for the thinner films. Detailed analysis indicates that the thinner films $(0.1 \mu \mathrm{m}<t<1.6 \mu \mathrm{m})$ have a similar type of in-plane texture as the $1.6 \mu \mathrm{m}$ film. Their texture structure can be also described by Fig. 4(b). In-plane texture is quantitatively evaluated using two physical variables: ${ }^{12}$ the half width at half maximum (HWHM) of the intensity peaks (in $\phi$ scans) and the volume fraction of the textured grains. Apparently, the two variables reflect the "degree" and the "amount" of textured grains, respectively. The HWHM is obtained by fitting the patterns from the azimuthal scans using Gaussian functions. The volume fraction of textured grains $V_{f}$ is defined as the ratio of integrated intensity from textured grains to that from the entire scattering volume of a film. The results of HWHM and $V_{f}$ are presented in Fig. 8. Both HWHM and $V_{f}$ appear to scale (approximately) linearly with film thickness $t$, especially as $t>0.4 \mu \mathrm{m}$. $V_{f}$ increases with increasing film thickness, and reaches more than $95 \%$ at $t=1.6 \mu \mathrm{m}$. This suggests that nearly all the grains are textured at such a thickness. HWHM, indicative of the angular spread of textured grains, decreases with increasing thickness. Its value approaches $10^{\circ}$ at $t=1.6 \mu \mathrm{m}$, indicating 


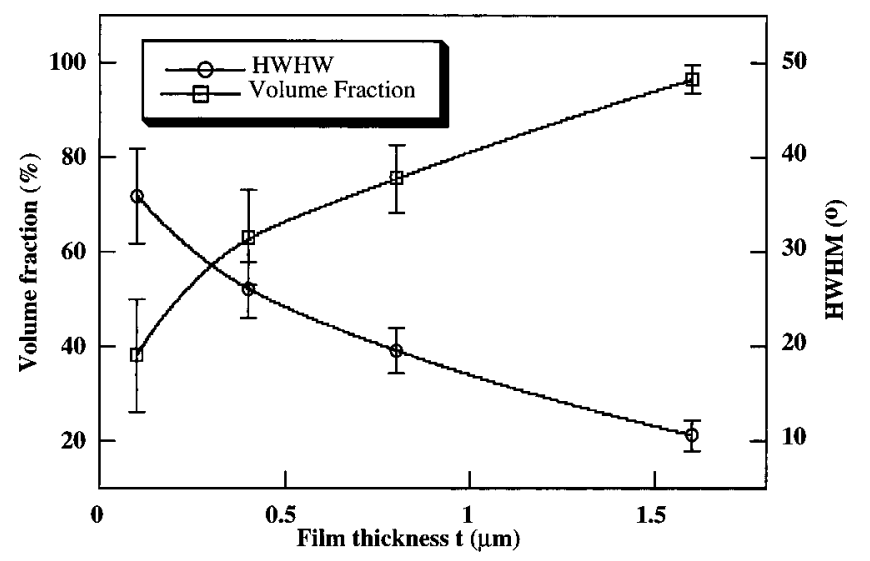

FIG. 8. Variations of the volume fraction of textured grains and HWHM (of peaks in intensity distributions), showing the evolution nature of the inplane texture.

that textured grains achieve a high degree of alignment at this thickness.

\section{Structure of the texture in thicker films}

Since the thicker films have the (111)-type out-of-plane texture, their in-plane texture is expected to differ from thinner films. This is evidenced by the azimuthal scans of the 4.5- $\mu \mathrm{m}$ film shown in Fig. 9, which reveals certain distinct features. First, the (200) reflection shows a uniform intensity distribution, as opposed to the cases of thinner films. Second, the intensity of the (211) reflection peaks at different azimuthal angles $(\phi)$. These variations are consistent with the fact that preferential growth direction now is [111] instead of [110]. In these thick films, few grains possess (200) planes perpendicular to the plane of film. This leads to the nearly uniform intensity distribution of the (200) reflection. Further-

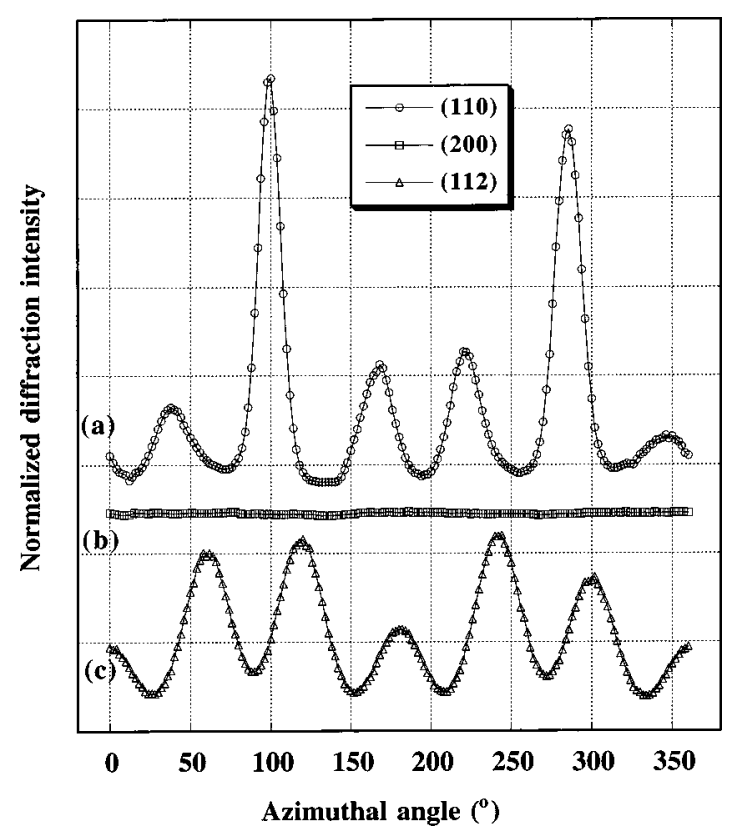

FIG. 9. Intensity distribution of the (110), (200), and (112) reflections from a $3.2-\mu \mathrm{m} \mathrm{Cr}$ film, obtained by azimuthal $\phi$ scan at a grazing incidence angle. (a)
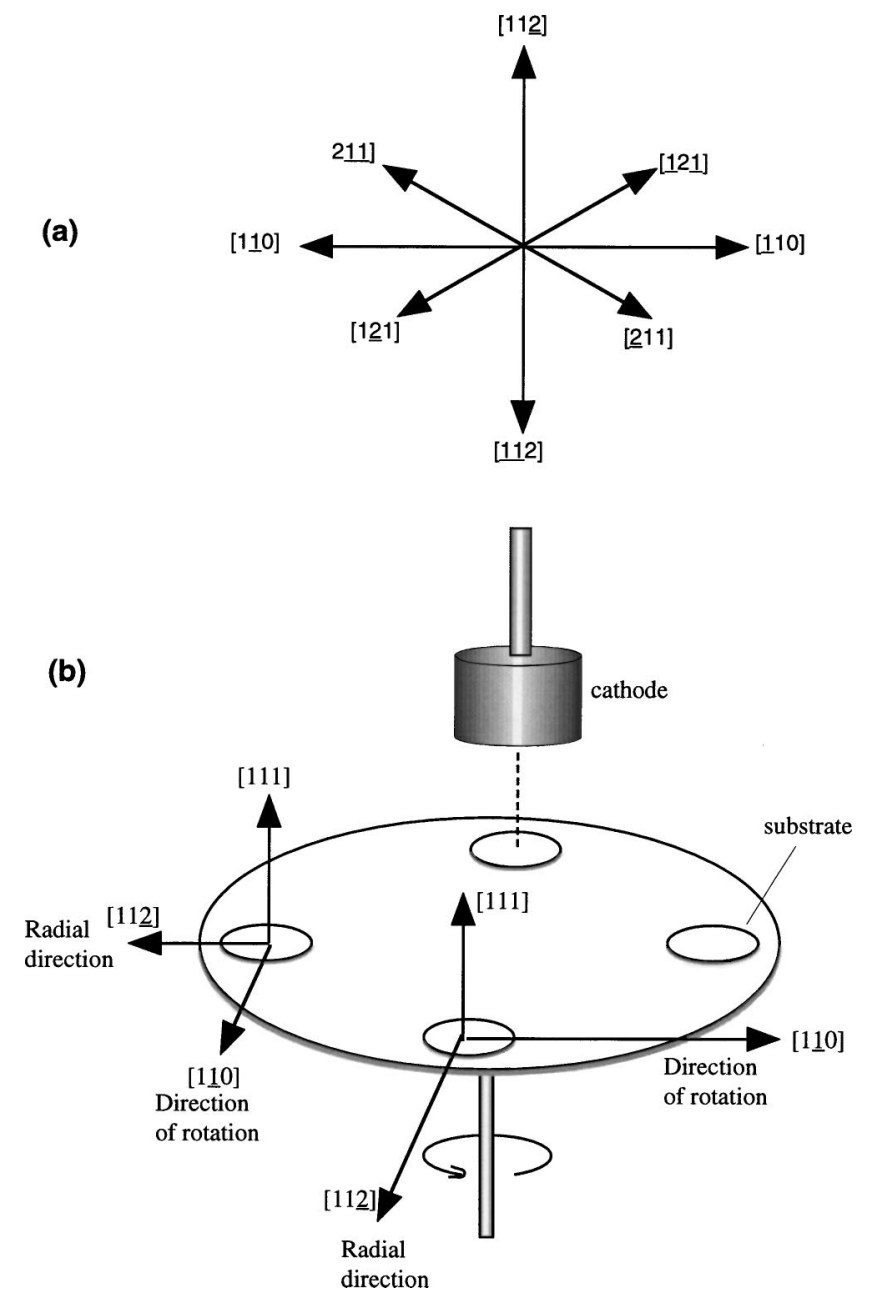

FIG. 10. (a) A [111] projection showing the relationships between several crystallographic orientations, and (b) a schematic drawing illustrating the correlation between the structure of texture in the thicker $\mathrm{Cr}$ films and the deposition geometry.

more, the presence of six peaks in the azimuthal scan of the (112) reflection in Fig. 9(c) agrees well with the (111) projection shown in Fig. 10(a). Based on these analyses, one can establish the correlation between the structure of texture in the thicker films and the deposition geometry, as shown in Fig. 10(b). The preferential orientation in the direction of platen rotation remains [110], and the one in the radial direction becomes [112]. This result is consistent with the (110) and (200) pole figures shown in Fig. 5(b).

\section{TEM observation and electron diffraction}

Figure 11 shows the plan view TEM results, where the microstructures in different depths of the 1.6- $\mu \mathrm{m} \mathrm{Cr}$ film are revealed. The micrograph in Fig. 11(a), taken from the layer $(\sim 500 \AA)$ near the film-substrate interface, represents the microstructure of the film at the very early stage of its deposition. The equiaxed, fine grains show little preferential orientation, as indicated by the electron diffraction pattern shown in Fig. 11(b). The micrograph in Fig. 11(c), taken from the top layer $(\sim 500 \AA)$, represents the microstructure of the film near the end of deposition. The microstructure is organized and anisotropic, consisting of elongated grains 
(a)



FIG. 11. Plan view TEM results of a 1.6- $\mu \mathrm{m} \mathrm{Cr}$ film. Note that (a) and (b) were taken from the top layer of the film, and (c) and (d) were taken from the layer adjacent to the film-substrate interface.

with aspect ratios as high as four. The grains, which elongate along the radial direction, are also aligned crystallographically. This is evidenced by its electron diffraction pattern in Fig. 11(d), on which a detailed analysis indicates that

(a) The preferential orientation in the direction of film growth is [110],

(b) The direction of grain elongation is [100], and [110].

(c) The in-plane direction normal to grain elongation is

These observations are consistent with the structure of texture deduced from x-ray diffraction studies.

Figure 12 presents the plan view TEM results of the $4.5-\mu \mathrm{m}$ film. The microstructure of its top layer $(\sim 500 \AA)$ is shown in Fig. 12(a). The grains are still elongated along the radial direction, but the aspect ratios become much smaller compared to the $1.6-\mu \mathrm{m}$ film. This suggests that the microstructure is less anisotropic for the thicker films. However, the grains are still well aligned crystallographically, as indicated by the electron diffraction pattern in Fig. 12(b). An analysis of the pattern leads to

(a) The preferential orientation in the direction of film growth is [111],

(b) The direction of grain elongation becomes [112], and

(c) The in-plane direction normal to grain elongation is still [110].

Again, these results are consistent with the structure of texture depicted in Fig. 10 obtained from x-ray diffraction studies. The micrograph in Fig. 12(c) was taken from a midlayer which is roughly $1.5 \mu \mathrm{m}$ from the film substrate. The micro-

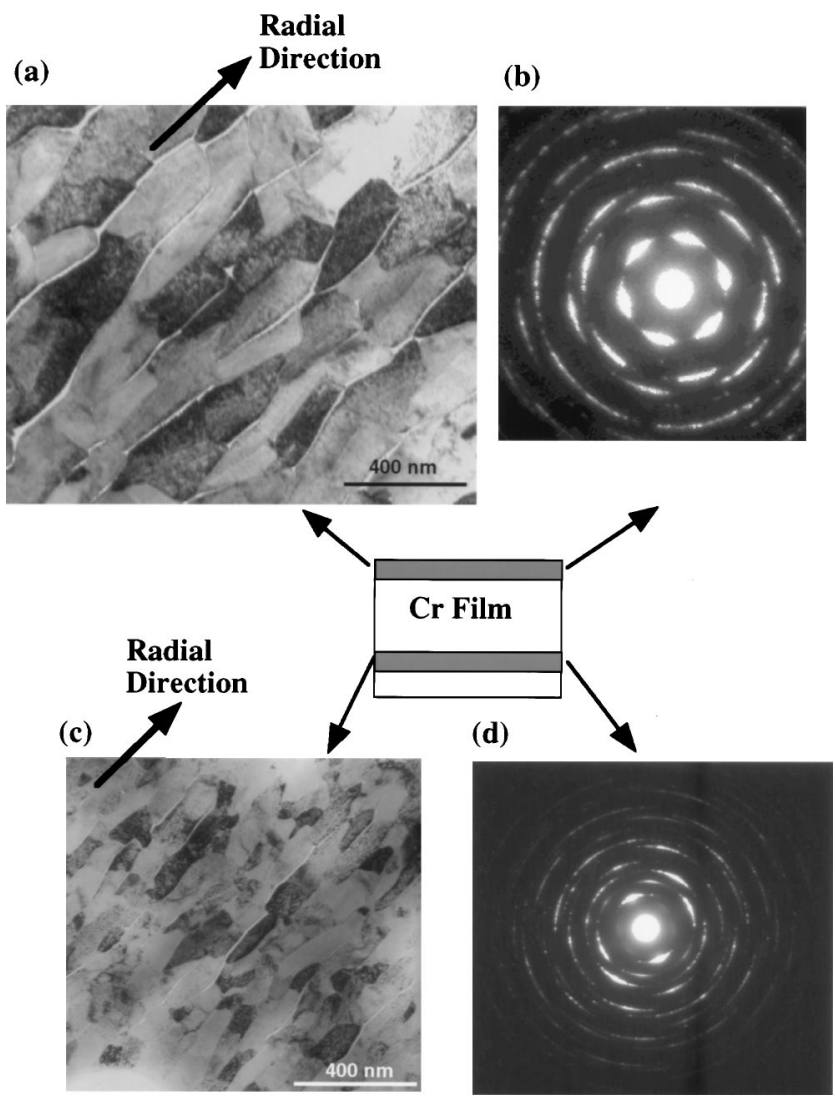

FIG. 12. Plan view TEM results of a 4.5- $\mu \mathrm{m}$ Cr film. Note that (a) and (b) were taken from the top layer of the film, and (c) and (d) were taken from a midlayer that is approximately $1.5 \mu \mathrm{m}$ from the film-substrate interface.

structure, not surprisingly, resembles that of the top layer of the 1.6- $\mu \mathrm{m}$ film. Figure 12(d) shows the electron diffraction pattern taken from the midlayer of the $4.5-\mu \mathrm{m}$ film, clearly pointing to the (110) out-of-plane texture. This further confirms the results by x-ray diffraction that texture changes from the (110) type to the (111) type in the thicker films.

\section{ANISOTROPIC STRESS IN THE Cr FILMS}

The stress in the $\mathrm{Cr}$ films is generally anisotropic and strongly depends on film thickness. As shown in Fig. 13, the in-plane stress state is characterized by two principal stresses: The stress in the radial direction $\sigma_{r}$ and the stress in the direction of platen rotation $\sigma_{p}$. For the very thin films, stress $\sigma_{r}$ is smaller than stress $\sigma_{p}$. Their differences become smaller as the film thickness increases within the range $0.1 \mu \mathrm{m}<t<0.4 \mu \mathrm{m}$. After a certain thickness $(t \sim 0.5 \mu \mathrm{m})$, stress $\sigma_{r}$ becomes gradually larger than $\sigma_{p}$. The stress anisotropy reaches its maximum at the thickness $t=1.6 \mu \mathrm{m}$, where $\sigma_{r}$ is several times larger than $\sigma_{p}$. Furthermore, the stresses $\sigma_{r}$ and $\sigma_{p}$ exhibit the different dependencies on film thickness. As the film thickness increases from 0.1 to 1.6 $\mu \mathrm{m}, \sigma_{p}$ decreases drastically while $\sigma_{r}$ increases slightly. As the film further thickens $(1.6 \mu \mathrm{m}<t<3.2 \mu \mathrm{m}), \sigma_{r}$ decreases rapidly while $\sigma_{p}$ shows little variation. Over these two ranges of film thicknesses, stresses $\sigma_{r}$ and $\sigma_{p}$ have displayed nearly reverse trends of variation. Such behavior appears to 


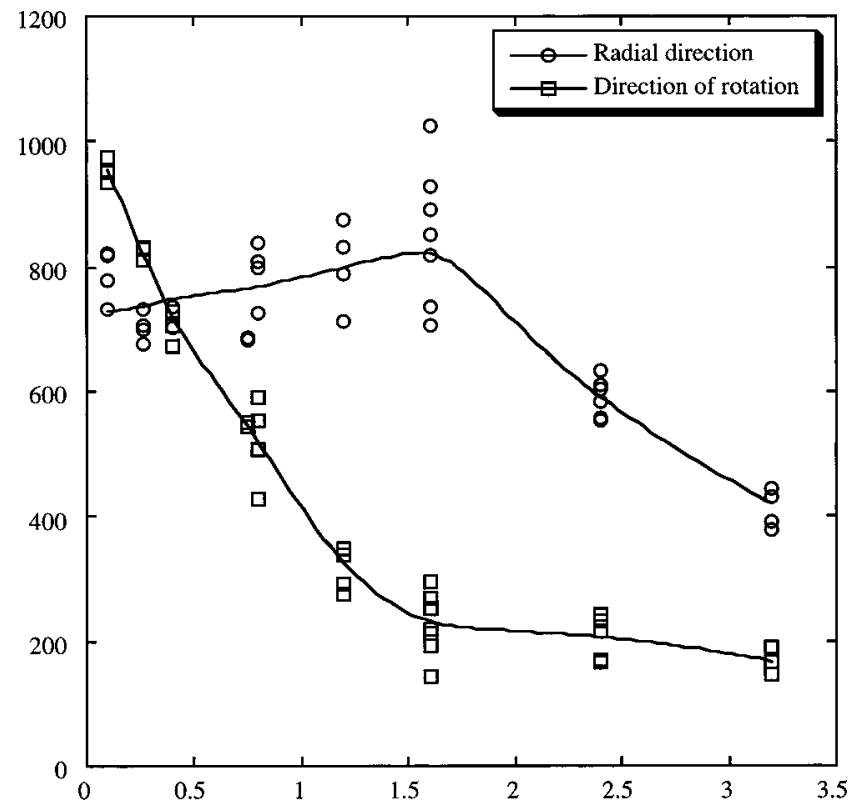

FIG. 13. Variation of the anisotropic stresses in $\mathrm{Cr}$ films as functions of film thickness.

be related to the microstructural evolution, the development of in-plane texture, and the texture change, as discussed in Sec. V.

\section{DISCUSSION}

The highest-stress anisotropy is observed at about $t$ $\sim 1.6 \mu \mathrm{m}$, which coincides with the occurrence of the utmost grain elongation (see Figs. 11, 12, and 14), along with the strongest in-plane texture. Furthermore, both principal stresses $\sigma_{r}$ and $\sigma_{p}$ in Fig. 13 show different trends of variations for films $t<1.6 \mu \mathrm{m}$ and $t>1.6 \mu \mathrm{m}$, respectively. These are the two ranges of film thickness, in which the Cr films develop different types of in-plane textures. Such observations suggest the links between the stress behaviors of the $\mathrm{Cr}$ films and their microstructural features, which are elucidated in the following three sections.

\section{A. Modulus effect associated with in-plane texture}

Generally, elastic properties of a crystal depend on crystallographic orientations. For a cubic crystal, the modulus in $[h k l]$ direction can be written as

$$
\frac{1}{E_{h k l}}=S_{11}-2\left[\left(S_{11}-S_{12}\right)-\frac{1}{2} S_{44}\right]\left(\alpha^{2} \beta^{2}+\alpha^{2} \gamma^{2}+\beta^{2} \gamma^{2}\right),
$$

where $S_{11}, S_{12}$, and $S_{44}$ are the elastic compliance and $\alpha, \beta$, and $\gamma$ are the directional cosines of $[h k l]$. Using $S_{11}$ $=0.28 ; S_{12}=-0.052$; and $S_{44}=0.97$ for $\mathrm{Cr}^{18}{ }^{18}$ one can get the following results:

$$
\begin{aligned}
& E_{100}=360 \mathrm{GPa} ; \quad E_{112}=300 \mathrm{GPa} ; \\
& E_{110}=270 \mathrm{GPa} ; \quad \text { and } E_{111}=250 \mathrm{GPa} .
\end{aligned}
$$

Now let us consider the textured $\mathrm{Cr}$ films as depicted in Figs. 4(b) and 10(b), respectively. For both types of in-plane textures, the radial direction coincides with the preferential orientations [100] or [112], which have the higher moduli.


FIG. 14. Scanning electron microscopy micrographs showing the surface structures of Cr films with different thickness. (a) $0.8 \mu \mathrm{m}$; (b) $1.6 \mu \mathrm{m}$; (c) $3.2 \mu \mathrm{m}$; and (d) $4.5 \mu \mathrm{m}$.

Therefore, the textured $\mathrm{Cr}$ films have the largest modulus in the radial direction. Such a modulus effect associated with in-plane texture could be a factor that contributes to the larger stress in radial direction than in the direction of platen rotation.

\section{B. Microstructural effect: Intercolumnar voids}

For the films within $0.1 \mu \mathrm{m}<t<1.6 \mu \mathrm{m}$, the different dependencies of $\sigma_{r}$ and $\sigma_{p}$ on film thickness could be interpreted based on the sources of generation and relaxation of tensile stress, namely, the atomic scale voids (mainly grain boundaries) and microscale voids (mainly intercolumnar voids). The voids, which exist between the elongated grains, align preferentially parallel to the radial direction. For the very thin films $(t<0.1 \mu \mathrm{m})$, the "voids" (if any) should be on an atomic scale, and behave like grain boundaries. The structural relaxations of these grain boundaries have been considered as the main source of tensile stress (often referred to as Hoffman stress ${ }^{14}$ ) in the deposited films of metals with low-atomic mobility, such as $\mathrm{Cr} .{ }^{19-22}$ This seems to explain that for the very thin $\mathrm{Cr}$ films $(0.1 \mu \mathrm{m}<t<0.4 \mu \mathrm{m})$, stress $\sigma_{p}$ is higher than stress $\sigma_{r}$. After a certain thickness, the film gradually develops microscale intercolumnar voids as a result of the shadowing effect. Such voids are so wide that atomic elastic couplings between neighboring grains are virtually impossible. Thus, they serve as the sources of stress relief, because these void regions cannot support stress. ${ }^{23}$ Apparently, the volume fraction of the intercolumnar voids increases as the film thickens, which contributes to the rapid drop in stress $\sigma_{p}$ within the range of $0.1 \mu \mathrm{m}<t<1.6 \mu \mathrm{m}$. On the other hand, the components of intercolumnar voids in the direction of platen rotation are negligibly small, which accounts for the little variation of $\sigma_{r}$ as a function of film thickness. 


\section{Stress-driven texture transition?}

As the out-of-plane texture changes from the (110) type to the (111) type, the preferential orientation in the radial direction changes to [112] from [100]. Correspondingly, the modulus of the $\mathrm{Cr}$ films is expected to decease in the radial direction, which contributes to the reduction in stress $\sigma_{r}$ (for $t>1.6 \mu \mathrm{m})$. Such a reduction in the elastic energy could be the driving force for the texture transition. This is plausible due to the following considerations:

(a) The grain elongation and the development of texture seems to reach saturation around $1.6 \mu \mathrm{m}$ (see Fig. 14). If the trend continues, these grains would grow into each other. Furthermore, there should be an up limit of the length of the grains, which is dictated by the surface diffusivity at the given deposition condition.

(b) As the film deposition proceeds, temperatures in the chamber and at the growing film surface increase, especially for the late growth stage of thick films. The atomic mobility and surface diffusion are enhanced, as indicated by the faceted surface structure shown by the thicker films (Fig. 14).

(c) At $t \sim 1.6 \mu \mathrm{m}$, the stress $\sigma_{r}$ reaches very high magnitudes. Given the high-stress state and the enhanced atomic ability/surface diffusion, it is plausible that the growth of the film searches for and transits to an energetically favorable configuration. The texture transition appears to serve such a purpose, as evidenced by the rapid decrease in $\sigma_{r}$ after $t$ $>1.6 \mu \mathrm{m}$. For metal films, changes of out-of-plane textures with thickness were previously reported in other systems. ${ }^{24,25}$ Similarly, the competition between surface energy and elastic strain energy was believed to be the cause for the texture turnover. ${ }^{26}$

\section{CONCLUSIONS}

Cr films prepared using dc magnetron sputter under the multiple-substrate geometry have been investigated in terms of their stress states and unique structural features. Generally, the stresses in these films are strongly anisotropic. The anisotropic stresses are characterized by two principal stresses. One is the stress in the radial direction $\left(\sigma_{r}\right)$ and the other is the stress in the direction of platen rotation $\left(\sigma_{p}\right)$. Both $\sigma_{r}$ and $\sigma_{p}$ are dependent on film thickness. The stress behavior, including both stress anisotropy and the film thickness dependence, is linked to the microstructural features, including the crystallographic textures. Generally, the $\mathrm{Cr}$ films develop well-organized microstructures, which consist of elongated grains separated with the preferentially aligned intercolumnar voids. The direction of grain elongation, consistently along certain crystallographic orientations, is related to the deposition geometry and coincides with the radial direction. The thinner films $(t<1.6 \mu \mathrm{m})$ develop (110) out-of-plane texture and the related in-plane texture. For thicker films, the out-of-plane texture becomes a (111) type and the related in-plane texture changes conformably. The correlation between the anisotropic stress and the film structures is discussed in terms of the modulus effect associated with inplane texture, the stress relief at intercolumnar voids, and the texture transition. Meanwhile, it is suggested that the texture transition may be driven by the reduction in elastic energy. a

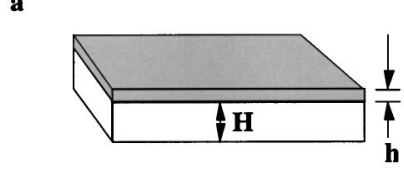

b

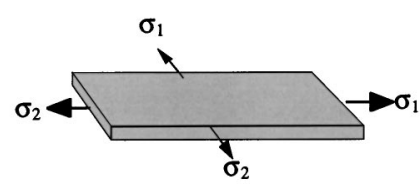

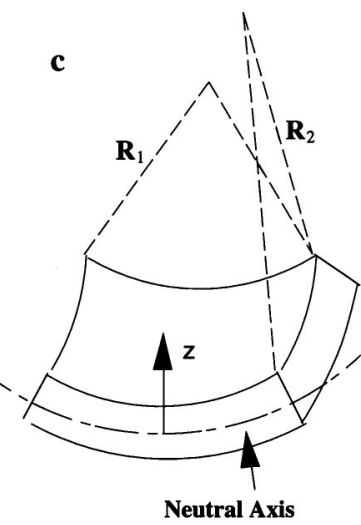

FIG. 15. The model for determination of anisotropic stress.

\section{ACKNOWLEDGMENTS}

The U.S. Army Research Office, Research Triangle Park, NC, supported this research work under Grant Nos. DAAG 55-98-1-0382 and DAAD 19-02-1-0335. The synchrotron radiation x-ray scattering experiments were conducted at Stanford Synchrotron Radiation Laboratory, a DOE supported facility. The authors would like to thank Dr. Jeff Hershberger (Argonne National Labs), Dr. Jay F. Whitacre (JPL, Caltech), and Ms. Beth Ann Rainey (IBM) for their help in x-ray scattering experiments.

\section{APPENDIX: DETERMINATION OF ANISOTROPIC STRESS}

For a film deposited onto a (110) or (111) Si single crystal, the isotropic residual stress in the film causes isotropic deformation of the substrate. ${ }^{27}$ The stress can be calculated using the well-known Stoney equation. ${ }^{28}$ In the case of stress anisotropy, the stress state in a thin film is described by two principal stresses $\sigma_{1}$ and $\sigma_{2}$. Other notations used in this work are shown in Fig. 15. For a film-substrate composite, the position of its neutral axis was considered by Davidenkov. ${ }^{29}$ Let $\delta$ be the shift of the neutral axis from the center plane of the substrate. It was given that

$$
\delta=\frac{E_{f}}{E_{s}} \frac{h}{2} \frac{\left(1+\frac{h}{H}\right)}{\left(1+\frac{h}{H} \times \frac{E_{f}}{E_{s}}\right)},
$$

where $E_{f}$ and $E_{s}$ are Young's modulus of film and substrate, respectively. This quantity is usually very small $(<1 \%$ of the substrate thickness). It is valid to assume that the neutral axis of the film-substrate composite locates at the center plane $z$ $=0$ of the substrate (as long as $h \ll H$ ). In fact, this is one of the underlying assumptions for the Stoney equation

$$
\sigma=\frac{E}{6(1-\nu)} \frac{H^{2}}{h} \frac{1}{R} .
$$

The principal strains at the $z$ plane in the substrate (Fig. 15) can be represented by

$$
\epsilon_{1}(z)=\frac{z}{R_{1}},
$$




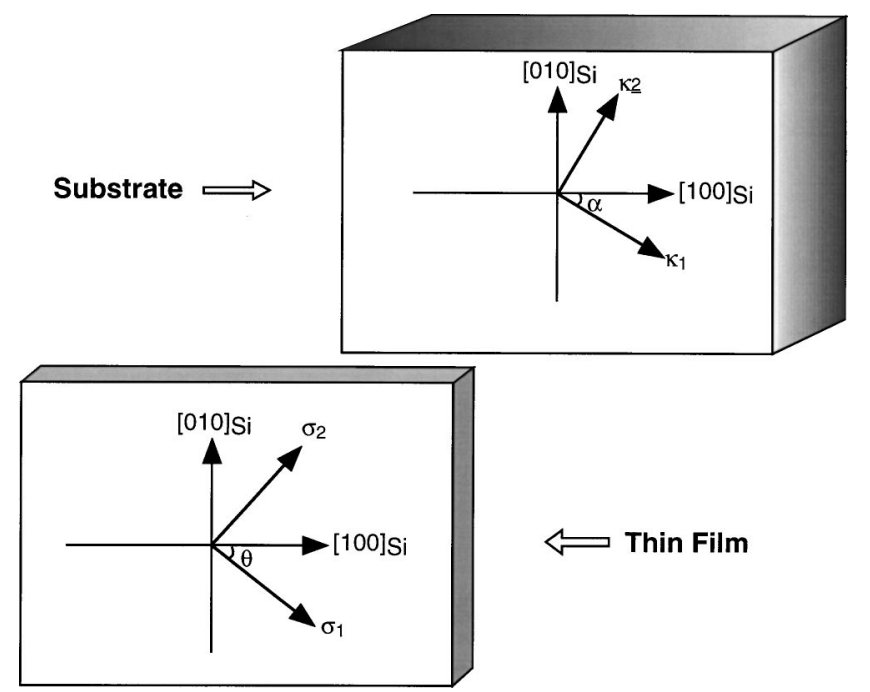

FIG. 16. The angle $(\theta)$ between the direction of principal stress $\sigma_{1}$ and the $[100]_{\mathrm{Si}}$ generally differs from the angle $(\alpha)$ between the direction of the principal curvature $\kappa_{1}$ and the $[100]_{\mathrm{Si}}$.

$$
\epsilon_{2}(z)=\frac{z}{R_{2}} \text {. }
$$

Consider a general case where the directions of principal curvatures do not coincide with the crystallographic directions $[100]_{\mathrm{Si}}$ and $[010]_{\mathrm{Si}}$. Under such a condition, the directions of the principal curvature may not necessarily reflect the directions of principal stresses in the film. In order to utilize the elasticity theory for cubic single crystal, one needs to perform tensor transformations. The notations used for the following analysis are shown in Fig. 16. Let $\epsilon_{x}(z)$, $\epsilon_{y}(z) \epsilon_{x y}(z)$, and $\epsilon_{z}(z)$ be the strain tensors and $\sigma_{x}(z)$, $\sigma_{y}(z) \sigma_{x y}(z), \sigma_{z}(z)$ be the stress tensors in the substrate with respect to the $x-y$ coordinate system, which coincides with $[100]_{\mathrm{Si}}$ and $[010]_{\mathrm{Si}}$. Then the strain tensor transformation leads to

$$
\begin{aligned}
& \epsilon_{x}(z)=\frac{\epsilon_{1}+\epsilon_{2}}{2}+\frac{\epsilon_{1}-\epsilon_{2}}{2} \cos 2 \alpha, \\
& \epsilon_{y}(z)=\frac{\epsilon_{1}+\epsilon_{2}}{2}-\frac{\epsilon_{1}-\epsilon_{2}}{2} \cos 2 \alpha, \\
& \epsilon_{x y}(z)=\frac{\epsilon_{2}-\epsilon_{1}}{2} \sin 2 \alpha,
\end{aligned}
$$

where $\epsilon_{1}$ and $\epsilon_{2}$ were represented by Eqs. (A3). The stressstrain relationships for single crystal can be given as

$$
\left[\begin{array}{c}
\epsilon_{x}(z) \\
\epsilon_{y}(z) \\
\epsilon_{z}(z) \\
\epsilon_{x y}(z)
\end{array}\right]=\left[\begin{array}{cccc}
s_{11} & s_{12} & s_{12} & 0 \\
s_{12} & s_{\mathrm{ss}} & s_{12} & 0 \\
s_{12} & s_{12} & s_{11} & 0 \\
0 & 0 & 0 & s_{44}
\end{array}\right]\left[\begin{array}{c}
\sigma_{x}(z) \\
\sigma_{y}(z) \\
0 \\
\sigma_{x y}(z)
\end{array}\right] .
$$

Combining Eqs. (A4) and (A5), one can obtain

$$
\sigma_{x}(z)=\frac{\epsilon_{1}(z)+\epsilon_{2}(z)}{2\left(s_{11}+s_{12}\right)}+\frac{\epsilon_{1}(z)-\epsilon_{2}(z)}{2\left(s_{11}-s_{12}\right)} \cos 2 \alpha,
$$

$$
\begin{aligned}
& \sigma_{y}(z)=\frac{\epsilon_{1}(z)+\epsilon_{2}(z)}{2\left(s_{11}+s_{12}\right)}-\frac{\epsilon_{1}(z)-\epsilon_{2}(z)}{2\left(s_{11}-s_{12}\right)} \cos 2 \alpha, \\
& \sigma_{x y}(z)=\frac{1}{s_{44}}\left[\epsilon_{2}(z)-\epsilon_{1}(z)\right] \sin 2 \alpha .
\end{aligned}
$$

These stress tensors can be further written as

$$
\begin{aligned}
\sigma_{x}(z)= & \frac{z}{2\left(s_{11}+s_{12}\right)}\left(\frac{1}{R_{1}}+\frac{1}{R_{2}}\right) \\
& +\frac{z}{2\left(s_{11}-s_{12}\right)}\left(\frac{1}{R_{1}}-\frac{1}{R_{2}}\right) \cos 2 \alpha, \\
\sigma_{y}(z)= & \frac{z}{2\left(s_{11}+s_{12}\right)}\left(\frac{1}{R_{1}}+\frac{1}{R_{2}}\right) \\
& -\frac{z}{2\left(s_{11}-s_{12}\right)}\left(\frac{1}{R_{1}}-\frac{1}{R_{2}}\right) \cos 2 \alpha, \\
\sigma_{x y}(z)= & \frac{z}{s_{44}}\left(\frac{1}{R_{1}}-\frac{1}{R_{2}}\right) \sin 2 \alpha .
\end{aligned}
$$

According to stress tensor transformation, the principal stresses at the $z$ plane of substrate are obtained as

$$
\begin{aligned}
\sigma_{1}(z)= & {\left[\left(\frac{1}{R_{1}}+\frac{1}{R_{2}}\right) \frac{1}{2\left(s_{11}+s_{12}\right)}\right.} \\
& +\left(\frac{1}{R_{1}}-\frac{1}{R_{2}}\right) \sqrt{\left.\frac{\cos ^{2} 2 \alpha}{4\left(s_{11}-s_{12}\right)^{2}}+\frac{\sin ^{2} 2 \alpha}{s_{44}^{2}}\right] z,} \\
\sigma_{2}(z)= & {\left[\left(\frac{1}{R_{1}}+\frac{1}{R_{2}}\right) \frac{1}{2\left(s_{11}+s_{12}\right)}\right.} \\
& \left.-\left(\frac{1}{R_{1}}-\frac{1}{R_{2}}\right) \sqrt{\frac{\cos ^{2} 2 \alpha}{4\left(s_{11}-s_{12}\right)^{2}}+\frac{\sin ^{2} 2 \alpha}{s_{44}^{2}}}\right] z .
\end{aligned}
$$

The momenta associated with substrate deformation can be obtained from Eqs. (A8) by integrating over the substrate thickness. Apparently, these momenta must be balanced by those associated with $\sigma_{1}$ and $\sigma_{2}$, the stress in the thin film, given as

$$
\begin{aligned}
& M_{1}^{f}=\left(\sigma_{1} h W\right) \frac{1}{2} H, \\
& M_{2}^{f}=\left(\sigma_{2} h L\right) \frac{1}{2} H .
\end{aligned}
$$

Based on the balance equations, one can obtain the equations correlating $\sigma_{1}$ and $\sigma_{2}$ to $R_{1}$ and $R_{2}$

$$
\begin{aligned}
\sigma_{1}= & \frac{1}{6}\left[\left(\frac{1}{R_{1}}+\frac{1}{R_{2}}\right) \frac{1}{2\left(s_{11}+s_{12}\right)}\right. \\
& +\left(\frac{1}{R_{1}}-\frac{1}{R_{2}}\right) \sqrt{\left.\frac{\cos ^{2} 2 \alpha}{4\left(s_{11}-s_{12}\right)^{2}}+\frac{\sin ^{2} 2 \alpha}{s_{44}^{2}}\right] \frac{H^{2}}{h},} \\
\sigma_{2}= & \frac{1}{6}\left[\left(\frac{1}{R_{1}}+\frac{1}{R_{2}}\right) \frac{1}{2\left(s_{11}+s_{12}\right)}\right. \\
& \left.-\left(\frac{1}{R_{1}}-\frac{1}{R_{2}}\right) \sqrt{\frac{\cos ^{2} 2 \alpha}{4\left(s_{11}-s_{12}\right)^{2}}+\frac{\sin ^{2} 2 \alpha}{s_{44}^{2}}}\right] \frac{H^{2}}{h} .
\end{aligned}
$$


The angle $\theta$ formed by $\sigma_{1}$ and $[100]_{\mathrm{Si}}$ can be determined by

$$
\tan 2 \theta=\frac{2\left(s_{11}-s_{12}\right)}{s_{44}} \tan 2 \alpha .
$$

If the [110] flat edge of the Si wafer is aligned with the radial direction (refer to the deposition geometry in Fig. 1), one has $\alpha=0$ and

$$
\begin{aligned}
& \sigma_{1}=\frac{1}{6\left(s_{11}^{2}-s_{12}^{2}\right)}\left(\frac{s_{11}}{R_{1}}-\frac{s_{12}}{R_{2}}\right) \frac{H^{2}}{h}, \\
& \sigma_{2}=\frac{1}{6\left(s_{11}^{2}-s_{12}^{2}\right)}\left(\frac{s_{11}}{R_{2}}-\frac{s_{12}}{R_{1}}\right) \frac{H^{2}}{h} .
\end{aligned}
$$

${ }^{1}$ V. Guilbaud-Massereau, A. Celerier, and J. Machet, Thin Solid Films 258, 185 (1995).

${ }^{2}$ A. K. Kulkarni and L. C. Chang, Thin Solid Films 301, 17 (1997).

${ }^{3}$ L. Tang and G. Thomas, J. Appl. Phys. 74, 5025 (1993).

${ }^{4}$ R. A. Miller and H. J. Holland, Thin Solid Films 298, 182 (1997).

${ }^{5}$ D. P. Tracy, D. B. Knorr, and K. P. Rodbell, J. Appl. Phys. 76, 2671 (1994).

${ }^{6}$ J. A. Szpunar, Mater. Res. Soc. Symp. Proc. 472, 45 (1997).

${ }^{7}$ S. L. Duan, J. O. Artman, and D. E. Laughlin, J. Appl. Phys. 67, 4913 (1990).

${ }^{8}$ Y. C. Feng, D. E. Laughlin, and D. N. Lambeth, J. Appl. Phys. 76, 7311 (1994).

${ }^{9}$ D. P. Ravipati, W. G. Haines, and J. L. Dockendorf, J. Vac. Sci. Technol. A 5, 1968 (1087).
${ }^{10}$ H. C. Tsai, B. B. Lai, and A. Eltoukhy, J. Appl. Phys. 71, 3579 (1992).

${ }^{11}$ O. P. Karpenko, J. C. Bilello, and S. M. Yalisove, J. Appl. Phys. 76, 4610 (1994).

${ }^{12}$ O. P. Karpenko, J. C. Bilello, and S. M. Yalisove, J. Appl. Phys. 82, 1397 (1997).

${ }^{13}$ C. V. Thompson and R. Carel, Mater. Sci. Eng., B 32, 211 (1995).

${ }^{14}$ R. W. Hoffman, Thin Solid Films 34, 185 (1976).

${ }^{15}$ H. Widischmann, CRC Crit. Rev. Solid State Mater. Sci. 17, 547 (1992).

${ }^{16}$ F. M. d'Heurle, Int. Mater. Rev. 34, 53 (1989).

${ }^{17}$ M. F. Doerner and W. D. Nix, CRC Crit. Rev. Solid State Mater. Sci. 14, 225 (1988).

${ }^{18}$ G. Simmons and H. Wang, Single Crystal Elastic Constants and Calculated Aggregate Properties: A Handbook, 2nd ed. (MIT, Cambridge, MA, 1971).

${ }^{19}$ M. Janda and O. Stefan, Thin Solid Films 112, 127 (1984).

${ }^{20}$ H. K. Pulker, Thin Solid Films 89, 191 (1982).

${ }^{21}$ R. Abermann and R. Koch, Thin Solid Films 129, 71 (1985).

${ }^{22}$ R. M. Fisher, J. Z. Duan, and A. G. Fox, Mater. Sci. Eng., A 117, 3 (1989).

${ }^{23}$ T. J. Vink, W. Walrave, J. L. C. Daams, A. G. Dirks, M. A. J. Somers, and K. J. A. van der Aker, J. Appl. Phys. 74, 988 (1993).

${ }^{24}$ P. K. Dutta and H. William, J. Phys. D 3, 839 (1974).

${ }^{25}$ U. C. Oh and J. H. Je, J. Appl. Phys. 74, 1692 (1993).

${ }^{26}$ J. H. Je, D. Y. Noh, H. K. Kim, and K. S. Liang, J. Appl. Phys. 81, 6126 (1997).

${ }^{27}$ W. A. Brantley, J. Appl. Phys. 44, 534 (1973).

${ }^{28}$ G. G. Stoney, Proc. R. Soc. London, Ser. A 32, 172 (1909).

${ }^{29}$ N. N. Davidenkov, Fiz. Tuerd. Tela (Leningrad) 2, 2919 (1960) [Sov. Phys. Solid State 2, 2595 (1961)]. 